\title{
MARKOV CHAIN ANALYSIS FOR LAND MOBILE SATELLITE MIMO CHANNELS
}

\author{
U. M. Ekpe, T. W. C. Brown, B. G. Evans \\ Centre for Communication Systems Research \\ University of Surrey \\ Guildford, Surrey GU2 7XH, United Kingdom
}

Keywords: LMS, MIMO, DCPM, Markov chain.

\begin{abstract}
In this paper, a Markov chain analysis is performed to uncover the duration statistics of a land mobile satellite system capable of switching between MIMO and Dual Circular Polarisation Multiplexing (DCPM). Capacities have been computed using synthetic and measurement campaign data to show that DCPM is a viable alternative to MIMO and depends on the cross-polar discrimination (XPD) ratio exceeding a certain minimum value.
\end{abstract}

\section{Introduction}

Land Mobile Satellite (LMS) multiple-input multiple-output (MIMO) systems using polarisation as a means to achieve channel independence have shown promising results in the quest for increased capacity without increasing the dimensions of the transmit and receive terminals [6]. Analysis has shown that due to the peculiar nature of the LMS channel, the traditional MIMO technique (in the case of the downlink, especially when the transmitting terminals are co-located on a single satellite) does not always provide up to twice the expected capacity improvement in comparison with the single-input single-output (SISO) channel as predicted in [1],[4]. Hence Dual Circular Polarisation Multiplexing (DCPM), which is a computationally simpler technique to MIMO, has been developed to provide good capacity improvements when the LMS channel condition is mostly line of sight (LOS) and with a high antenna cross-polar discrimination (XPD).

Most LMS systems generally time-share between LOS and non line of sight (NLOS) periods but with a greater percentage of time spent in the former. A Markov chain analysis is necessary to obtain the probabilistic time spent in each of these periods so as to determine when the use of DCPM would be more beneficial than MIMO and vice versa for LMS systems. This paper is organised as follows: Section 2 describes the MIMO channel model and builds the MIMO channel matrix from synthetic and measurement campaign data. Section 3 uses two metrics for characterising the propagation channel. These metrics are the channel ergodic capacity and the receiving antenna cross-polar discrimination ratio. In section 4, DCPM is introduced and the formula for computing its channel capacity is derived. Achievable capacities using MIMO and DCPM are then compared using synthetic channel data and measurement campaign data. Finally, section 5 contains a Markov chain analysis of data from an emulated low elevation dual polarised LMS MIMO system.

\section{MIMO channel model}

The linear relationship for the narrowband time-invariant MIMO radio channel having $n$ transmitting and $m$ receiving antennas is given by:

$$
\boldsymbol{y}=\mathbf{H} \boldsymbol{x}+\boldsymbol{n}
$$

where $\boldsymbol{y} \in C^{m}$ is the received signal vector, $\boldsymbol{x} \in C^{n}$ is the transmitted vector, $\mathbf{H}$ is an $m \times n$ complex channel gain matrix, and $\boldsymbol{n}$ represents the complex valued additive white Gaussian noise which has zero-mean and equal variance.

\subsection{Measured channel data}

In this study, the narrowband LMS MIMO channel data (contained in matrix $\mathbf{H}$ ) was extracted from stored channel impulse response obtained from a wideband measurement campaign [6] in which the satellite was emulated using a hill top mounted platform transmitting with dual circular polarised antennas to a vehicular mobile receiver driven around different environments in Guildford, UK.
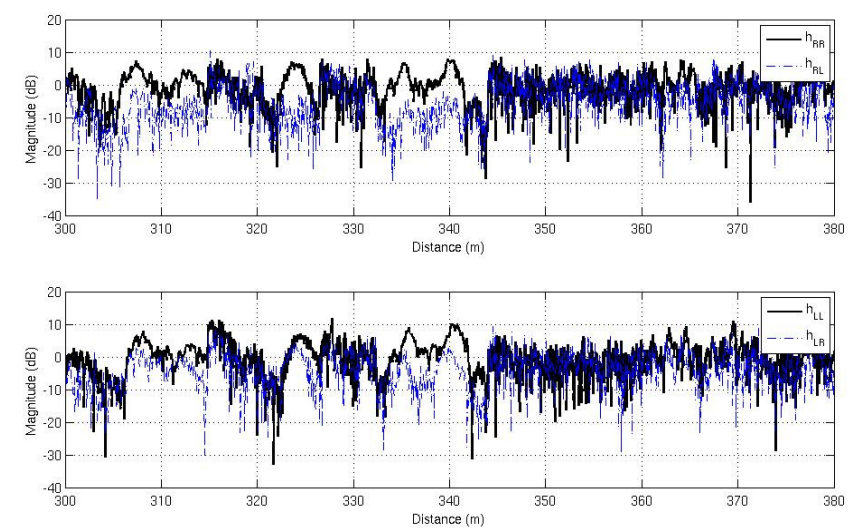

Figure 1: Received co- and cross-polar signal on a measurement run 
Figure 1 shows the normalised co- and cross-polar received power on the two orthogonally polarised antennas. The MIMO channels are $h_{R R}, h_{R L}, h_{L L}$ and $h_{L R} . h_{R R}$ represents the co-polar channel from a right hand circular polarised (RHCP) transmitting antenna to an RHCP receiving antenna. $h_{R L}$ represents the cross-polar channel from an RHCP transmitter to a left hand circular polarised (LHCP) receiver. The remaining two channels follow the same nomenclature but with respect to the LHCP antenna as shown in Figure 2.

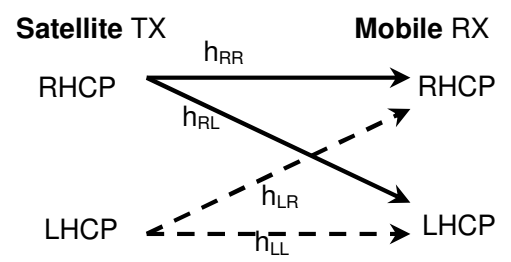

Figure 2: Schematic of the dual polarised LMS MIMO channel

\subsection{Synthetic channel data}

Experimental channel data was generated based on the phenomenon reported in [6] and [8] that the cross-polar components, $h_{R L}$ and $h_{L R}$, should ideally be zero but are never quite the case in practical dual polarised systems. Hence for the synthetic channel data analysed in this paper, these components were made to be considerably weaker than the co-polar components depending on the receiving antennas XPD (represented by $M$ in Figure 3) which were varied from $3 \mathrm{~dB}$ to $18 \mathrm{~dB}$ in order to determine their effects on system performance. Thus, the two co-polar components were normalised to the same magnitude but made to have different randomly distributed phases while the cross-polar components were generated in the same way and attenuated based on the available XPD.

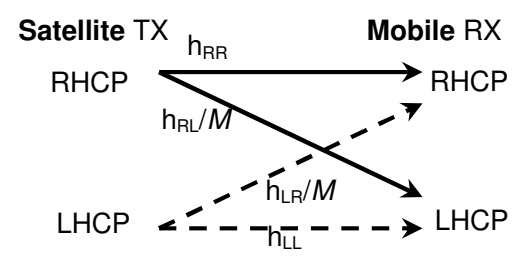

Figure 3: Schematic of synthetic dual polarised LMS MIMO channel

\section{Channel characterisation metrics}

Channel characterisation metrics are necessary for selecting the optimal mode of operation for communication systems. Among the commonly used metrics are the channel ergodic capacity and the receiving antenna cross-polar discrimination ratio. This paper will use these two metrics in comparing the performance of DCPM and MIMO for LMS systems.

\subsection{Channel ergodic capacity}

The capacity of the single-input single-output system was derived by Shannon [5]as:

$$
C=\log _{2}\left(1+\rho|h|^{2}\right) \quad \mathrm{b} / \mathrm{s} / \mathrm{Hz}
$$

where $\rho$ is the signal to noise ratio (SNR) at the receive antenna and $h$ is the normalised gain of the channel. From Equation (2), the well known MIMO capacity was then derived in [4] as:

$$
C=\log _{2}\left[\operatorname{det}\left(\mathbf{I}_{n}+\frac{\rho}{N} \mathbf{H H}^{H}\right)\right] \mathrm{b} / \mathrm{s} / \mathrm{Hz}
$$

where $\mathbf{I}_{n}$ is an $n \times n$ identity matrix, $N$ is the number of transmit antennas and $\mathbf{H}^{H}$ is the Hermitian transpose of $\mathbf{H}$. In terms of the Eigenvalues $\left(\lambda_{i}\right)$ of $\mathbf{H} \mathbf{H}^{H}$, MIMO capacity for the wireless channel is given in [11] as:

$$
C=\sum_{i=1}^{m} \log _{2}\left(1+\frac{\rho}{N} \lambda_{i}\right) \quad \mathrm{b} / \mathrm{s} / \mathrm{Hz}
$$

For the analysis in this paper, the capacity of the dual polarised LMS MIMO channel will then be derived from Equation (4) while the DCPM capacity will be derived based on the signal to noise plus interference ratios (SINRs) obtained after interference mitigation procedures.

\subsection{Cross polar discrimination ratio}

The cross-polar discrimination ratio (XPD) measures the level of depolarisation (i.e. the extent at which signals transmitted on one polarisation, like RHCP, is scattered into the opposite polarisation, like LHCP) a signal suffers as it propagates from the transmitter to the receiver [9]. The XPD also gives an indication of the antenna's ability to reject orthogonally polarised signals. Using the nomenclature developed for our dual polarised LMS MIMO system, the XPD in decibels can be obtained from:

$$
X P D_{1}=20 \log \frac{h_{R R}}{h_{R L}}
$$

and

$$
X P D_{2}=20 \log \frac{h_{L L}}{h_{L R}}
$$

It has been reported in [10] that $X P D_{1}=X P D_{2}$ in many cases.

\section{Dual Circular Polarisation Multiplexing}

Dual Circular Polarisation Multiplexing (DCPM) is a technique to optimally combine two orthogonally polarised channels for increased capacity when the LMS channel condition is mainly LOS and with a high XPD. For our twotransmit two-receive dual polarised system shown in Figure 2, this set up allows for the co-location of the antennas at both link ends even with the channels experiencing highly correlated fading. DCPM was first introduced in [2] and the channel weighting requirements explained therein. The schematic diagram in Figure 4 shows where the phase weights are applied for interference mitigation. These phase weights are then derived in the equations that follow using the method reported in [9]. 


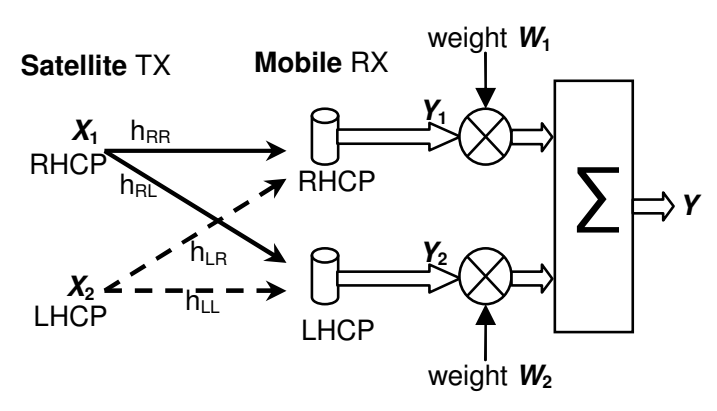

Figure 4: Interference mitigation and channel weighting

From Equation (1), the received signal vector in Figure 4 is given as:

$$
\begin{aligned}
& y_{1}=x_{1} h_{R R}+x_{2} h_{L R} \\
& \text { and } \\
& y_{2}=x_{1} h_{R L}+x_{2} h_{L L}
\end{aligned}
$$

The combiner's output is then given by:

$$
y=y_{1} w_{1}+y_{2} w_{2}
$$

Substituting appropriately, we have

$$
\begin{aligned}
& y=\left(x_{1} h_{R R}+x_{2} h_{L R}\right) w_{1}+\left(x_{1} h_{R L}+x_{2} h_{L L}\right) w_{2} \\
& =x_{1}\left(h_{R R} w_{1}+h_{R L} w_{2}\right)+x_{2}\left(h_{L R} w_{1}+h_{L L} w_{2}\right)
\end{aligned}
$$

The coefficient of $x_{1}$ is forced to zero while the coefficient of $x_{2}$ is forced to one to obtain the signal transmitted from the RHCP antenna. The opposite is carried out to obtain LHCP signals. Using the synthetic data and incorporating the XPD (represented by $M$ ), we denote the weightings for obtaining RHCP as $w_{1 A}$ and $w_{2 A}$ while the weightings for obtaining LHCP are $w_{1 B}$ and $w_{2 B}$, we then have:

$$
\begin{aligned}
& h_{R R} w_{1 A}+\frac{h_{R L}}{M} w_{2 A}=1, \\
& \frac{h_{L R}}{M} w_{1 A}+h_{L L} w_{2 A}=0
\end{aligned}
$$

and

$$
\begin{aligned}
& h_{R R} w_{1 B}+\frac{h_{R L}}{M} w_{2 B}=0, \\
& \frac{h_{L R}}{M} w_{1 B}+h_{L L} w_{2 B}=1
\end{aligned}
$$

Solving for the phase weights, we obtain:

$$
\begin{aligned}
& w_{1 A}=\frac{h_{L L} M^{2}}{h_{R R} h_{L L} M^{2}-h_{R L} h_{L R}}=\frac{h_{L L} M}{\Delta \mathrm{H}} \\
& w_{2 A}=\frac{h_{L R} M}{h_{R L} h_{L R}-h_{R R} h_{L L} M^{2}}=\frac{-h_{L R}}{\Delta \mathrm{H}}
\end{aligned}
$$

$$
w_{1 B}=\frac{h_{R L} M}{h_{R L} h_{L R}-h_{R R} h_{L L} M^{2}}=\frac{-h_{R L}}{\Delta \mathrm{H}}
$$

$$
w_{2 B}=\frac{h_{R R} M^{2}}{h_{R R} h_{L L} M^{2}-h_{R L} h_{L R}}=\frac{h_{R R} M}{\Delta \mathrm{H}}
$$

where $\Delta H=h_{R R} h_{L L} M-\frac{h_{R L} h_{L R}}{M}$

It should be noted that $M$ is already embedded in the obtained channel impulse response function in the case of measurement campaign data.

The capacity for DCPM can then be computed from:

$$
C_{D C P M}=\log _{2}\left(1+\operatorname{SINR}_{1}\right)+\log _{2}\left(1+\operatorname{SINR}_{2}\right)
$$

The SINRs in (18) are:

$$
\operatorname{SINR}_{1}=\frac{\left|h_{R R} w_{1 A}+\frac{h_{R L}}{M} w_{2 A}\right|^{2}}{\left|\frac{h_{L R}}{M} w_{1 A}+h_{L L} w_{2 A}\right|^{2}+\sigma_{n}^{2}}
$$

and

$$
\operatorname{SINR}_{2}=\frac{\left|\frac{h_{L R}}{M} w_{1 B}+h_{L L} w_{2 B}\right|^{2}}{\left|h_{R R} w_{1 B}+\frac{h_{R L}}{M} w_{2 B}\right|^{2}+\sigma_{n}^{2}}
$$

In Equations (19) and (20), the noise floor, $\sigma_{n}^{2}$ has been introduced for a fair capacity comparison of DCPM with MIMO and is given by:

$$
\sigma_{n}^{2}=\frac{\left|h_{R R}\right|^{2}}{S N R}
$$

An expansion of Equation (3) gives the MIMO capacity as:

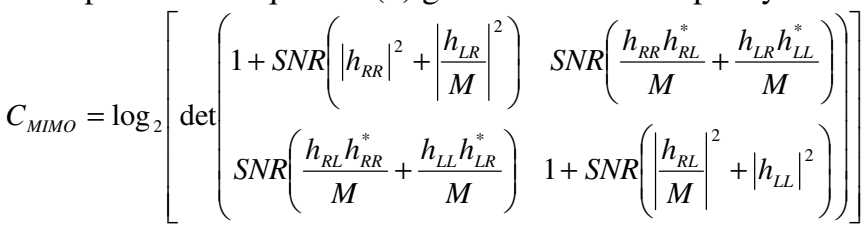

$$
\begin{aligned}
& =\log _{2}\left[\begin{array}{c}
(1+S N R)\left(\left|h_{R R}\right|^{2}+\left|\frac{h_{L R}}{M}\right|\right)(1+S N R)\left(\left|\frac{h_{R L}}{M}\right|^{2}+\left|h_{L L}\right|^{2}\right) \\
-S N R^{2}\left(\frac{h_{R L} h_{R R}^{*}}{M}+\frac{h_{L L} h_{L R}^{*}}{M}\right)\left(\frac{h_{R R} h_{R L}^{*}}{M}+\frac{h_{L R} h_{L L}^{*}}{M}\right)
\end{array}\right]
\end{aligned}
$$

In order to compare achievable capacities of DCPM and MIMO, Equations (18) and (22) were respectively used with 
the synthetic channel data at a realistic SNR of $10 \mathrm{~dB}$. The results of these capacities for different values of $M$ are shown in Figure 5. Notice how DCPM capacity improves as $M$ increases.

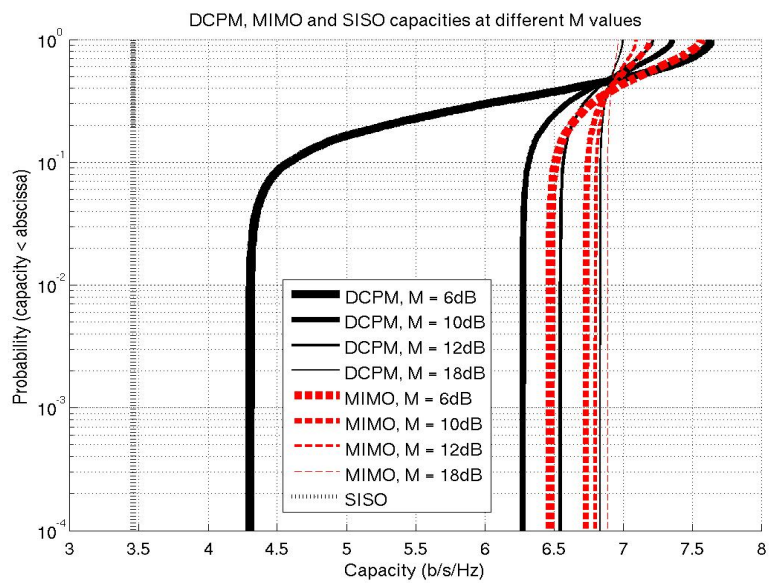

Figure 5: CDF plots of DCPM, MIMO and SISO capacities computed using synthetic data

For the measurement data, two sections are chosen for capacity comparison. The first being a section in which the XPD is in the order of $12 \mathrm{~dB}$ while the second section has an XPD of about $1 \mathrm{~dB}$. These sections correspond to periods of clear LOS and NLOS/heavily shadowed LOS respectively. A plot of the achievable capacities is shown in Figure 6.

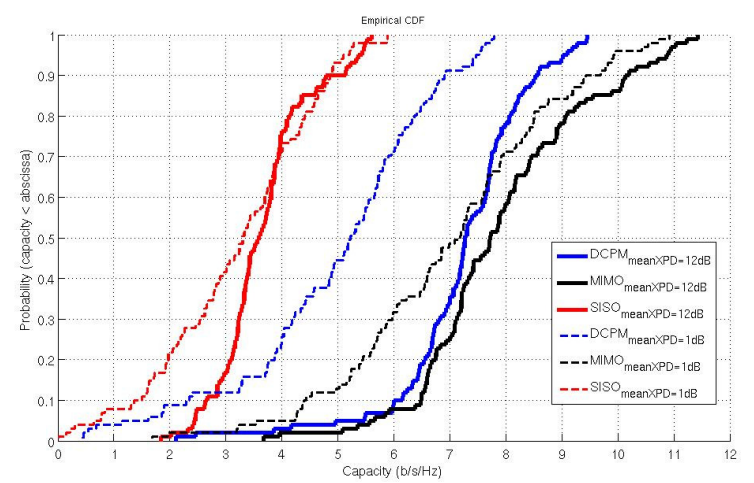

Figure 6: CDF plots of DCPM, MIMO and SISO capacities computed from measured data

Observe in that DCPM achieves a capacity within $0.5 \mathrm{~b} / \mathrm{s} / \mathrm{Hz}$ of MIMO when the XPD is $12 \mathrm{~dB}$ at $50 \%$ outage. This impressive capacity increase with DCPM is achieved using only interference mitigation applied at the mobile terminal as compared with MIMO where the equation used (Equation (22)) assumes that the transmitter (based on the satellite) has perfect knowledge of the channel. The perfect channel state information (CSI) is obtained by the receiver feeding back instantaneous channel gain to the transmitter. This method if attempted practically (assuming the feedback delay is not excessive) is bound to deplete available spectrum and complicate system architecture.
Figure 6 also shows that MIMO performs reasonably well when the XPD is low. Since this is the case it may require designing a system to switch from uninformed MIMO (no CSI feedback) when XPD is low (which corresponds to NLOS situations) to DCPM when the XPD is high. To achieve this, it is worth exploring the duration statistics of when the XPD is above or below a threshold value, hence the need for a Markov chain analysis.

\section{Markov chain analysis}

Several authors including [3],[6],[7] have used two to five state Markov processes to model the variation in the received signal for LMS systems. In this paper, a two state Markov model is used to show when the channel condition is favourable for either MIMO or DCPM implementation. The factor governing the switching process is the XPD which is set at a threshold level of $10 \mathrm{~dB}$ based on capacity improvements observed with synthetic data simulations (See Figure 5). Below this level, the channel favours MIMO implementation for capacity increase while above this level, using DCPM would be more beneficial. The two state Markov model is shown in Figure 7 while the $2 \times 2$ transition matrix and the 2-state vectors derived from narrowband data obtained from measured data is shown in Table 1.

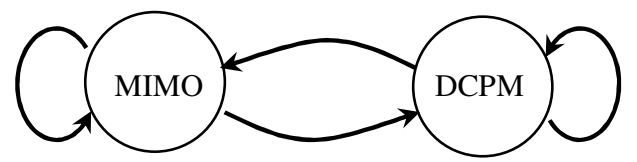

Figure 7: State transition diagram of a Markov chain

\begin{tabular}{|c|c|c|}
\hline \multicolumn{2}{|c|}{ Probability of state change } & $\begin{array}{c}\text { State probability } \\
\text { matrix }\end{array}$ \\
\hline $\mathrm{Pmm}=0.9011$ & $\mathrm{Pmd}=0.0988$ & $\mathrm{MIMO}=0.8743$ \\
\hline $\mathrm{Pdm}=0.6874$ & $\mathrm{Pdd}=0.3128$ & $\mathrm{DCPM}=0.1257$ \\
\hline
\end{tabular}

Table 1: Markov state and transition matrix for measured narrowband data

The probabilities of state change in Table 1 are computed following [3] from:

$$
P_{i j}=N_{i j} / N_{i}
$$

where $P_{i j}$ is the probability of changing from state $i$ state $j, N_{i j}$ is the number of transitions from state $i$ state $j$, and $N_{i}$ is the number of instances of state $i$. The state probability matrix is obtained using:

$$
S_{i}=N_{i} / N_{t}
$$

where $S_{i}$ is the probability of having state $i, N_{i}$ is the number of instances of state $i$, and $N_{t}$ is the total number of instances of all states. Thus the entries in Table 1 are interpreted as follows:

- Pmm represents the probability of remaining in a state favourable for MIMO implementation.

- Pmd represents the probability of changing from a MIMO-favourable to a DCPM-favourable state. 
- Pdm represents the probability of changing from a DCPM-favourable to a MIMO-favourable state.

- Pdd represents the probability of remaining in a state favourable for DCPM implementation.

It can be observed from Table 1 that a greater time-share was spent in conditions favourable for MIMO. This is because the data used in this analysis was obtained from a measurement campaign done at very low elevations ranging from $7^{\circ}$ to $18^{\circ}$. An inspection of the measurement environment and terrain data base revealed that at such low elevation, the propagation path from the hill-top mounted transmitter (emulating the satellite) to the vehicular mobile receiver was mostly NLOS.

\section{Conclusion}

This paper has presented a Markov chain analysis of dual polarised LMS MIMO channel data and built a two-state model for estimating the duration statistics of a MIMO and DCPM-capable system. The cross-polar discrimination ratio was used as the controlling factor for state transition and its value was derived based on DCPM and MIMO capacity estimates from synthetic and measured channel data. Future work is to be performed based on new measurement data obtained at higher elevation angles to validate the performance of DCPM.

\section{Acknowledgements}

The authors would like to thank the Satellite Network of Excellence (SatNEx) for helpful discussions on LMS MIMO channel modelling.

\section{References}

[1] L. M Correia, Ed., Mobile Broadband Multimedia Networks: Techniques, Models and Tools for 4G, Academic Press, London, UK, 2006.

[2] U. M. Ekpe, T. W. C. Brown, B. G. Evans, "Unleashing the Polarisation Domain for Land Mobile Satellite MIMO Systems," to appear in Proceedings of the $3^{\text {rd }}$ European Conference on Antennas and Propagation (EuCAP 09), Mar 2009.

[3] F. P. Fontan and P. M Espineira, Modeling the Wireless Propagation Channel - A Simulation Approach with MATLAB, John Wiley, West Sussex, UK, 2008.

[4] G. J. Foschini and M. J. Gans, "On Limits of Wireless Communications in a Fading Environment when using Multiple Antennas," Wireless Personal Communications, vol. 6, no. 3, pp. 311-335, 1998.

[5] D. Gesbert, M. Shafi, D. Shiu, P. J. Smith, and A. Naguib, "From Theory to Practice: An Overview of MIMO Space-Time Coded Wireless Systems," IEEE Journal on Selected Areas in Communications, vol. 21, no. 3, pp. 281-302, Apr. 2003.

[6] P. R. King, "Modelling and Measurement of the Land Mobile Satellite MIMO Radio Propagation Channel," PhD Thesis, University of Surrey, Guildford, UK, Apr. 2007.
[7] E. Lutz, D. Cygan, M. Dippold, F. Dolainsky, and W. Papke, "The Land Mobile Satellite Communication Channel - Recording, Statistics and Channel Model," IEEE Transactions on Vehicular Technology, vol. 40, no. 2, pp. 375-386, May 1991.

[8] C. Oestges, B. Clerckx, M. Guillaud, and M. Debbah, "Dual-Polarized Wireless Communications: From Propagation Models to System Performance Evaluation," IEEE Transactions on Wireless Communications, vol. 7, no. 10, pp. 4019-4031, Oct. 2008.

[9] S. R. Saunders, Antennas and Propagation for Wireless Communication Systems, John Wiley, West Sussex, UK, 1999.

[10]W. L. Stutzman, Polarization in Electromagnetic Systems. Boston: Artech House, 1992.

[11]E. Telatar, "Capacity of Multi-antenna Gaussian Channels," European Transactions on Telecommunications, vol. 10, no. 6, pp. 585-595, 1999. 